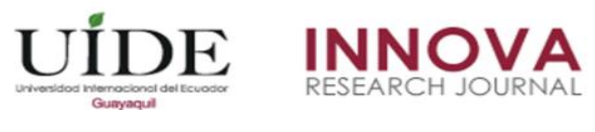

INNOVA Research Journal, ISSN 2477-9024

(Agosto, 2018). Vol. 3, No.8.1 pp. 16-32

DOI: https://doi.org/10.33890/innova.v3.n8.1.2018.752

URL: http://revistas.uide.edu.ec/index.php/innova/index

Correo: innova@uide.edu.ec

\title{
Las TIC como elemento catalizador en la generación de nuevas formas de negocios y emprendimientos en Ecuador a través del uso de los infocentros comunitarios
}

\section{ICT as a catalyst in the generation of new business forms and enterprises in Ecuador through the use of community infocenters.}

Esthela Maribel Cabezas

Hugo Christiam Fiallos Aguilar

Universidad Internacional del Ecuador, Ecuador

Autor para correspondencia: escabezasmo@uide.edu.ec, hufiallosag@uide.edu.ec

Fecha de recepción: 20 de julio de 2018 - Fecha de aceptación: 30 de agosto de 2018

Resumen: El avance tecnológico de un país, impulsa su desarrollo social y su crecimiento económico. Sobre todo si observamos que la sociedad en su conjunto va adquiriendo nuevas formas de relacionamiento, tanto en el ámbito personal, familiar y también en la forma de hacer negocios. A nivel latinoamericano, la mayoría de indicadores muestran una situación dispar en comparación con los países del primer mundo en materia de adopción y acceso a las TIC (Tecnologías de la Información y Comunicación), cuyo nivel de apropiación tiene una relación directamente proporcional con su competitividad y productividad. Particularmente en Ecuador, esta situación se torna evidente, si analizamos los principales indicadores asociados al acceso a TIC y su relación en el ámbito de negocios. Los infocentros comunitarios constituyen puntos de encuentro con las TIC, sobre todo en zonas rurales, que permiten a la ciudadanía descubrir oportunidades de desarrollo para generar emprendimientos, gracias a las capacitaciones en temas que fomentan y promueven la generación de emprendimientos. La presente investigación está orientada a establecer la situación actual de Ecuador en materia de acceso y adopción de las TIC y su incidencia en los emprendimientos sobre todo en los sectores rurales, a través del uso de los infocentros comunitarios.

Palabras Claves: TIC; emprendimiento; infocentro; negocios

\begin{abstract}
The technological advance of a country, promotes its social development and economic growth. Especially if we observe that society as a whole is acquiring new forms of relationship, both in the personal, family and also in the way of doing business. At the Latin American level, most indicators show a different situation compared to first world countries in terms of adoption and access to ICT (Information and Communication Technologies), whose level of ownership is directly proportional to their competitiveness and productivity. Particularly in Ecuador, this situation becomes evident, if we analyze the main indicators associated with access to ICT and its relationship in the field of business. Community infocenters are points of contact with ICTs, especially in rural areas, which allow citizens to discover development opportunities to generate entrepreneurship, thanks to training in topics that promote and promote the generation of ventures. This research is aimed at establishing the current situation of Ecuador in terms of access and adoption of ICT and its impact on the enterprises, especially in rural sectors, through the use of community infocentros.
\end{abstract}

Key Words: ICT; entrepreneurship; infocenter; business 


\section{Introducción}

El emprendimiento puede ser entendido como el comportamiento del ser humano para asumir riesgos y generar nuevos negocios. Y precisamente el emprendimiento se constituye en la actualidad en uno de los escenarios en donde mayor incidencia tienen las TIC, favoreciendo esquemas de globalización, acortando distancias, llegando a mercados diversos.

En cuanto al impacto de las TIC sobre el emprendimiento, diversos estudios han demostrado que la forma de emprender varía en el tiempo, conforme el avance y evolución propio de la sociedad. Concluyendo además que tanto las TIC y la desregulación de los mercados, favorecen la creación de nuevos emprendimientos.

El objetivo de la presente investigación es establecer la situación actual de Ecuador en materia de acceso y adopción de las TIC y su incidencia a través de los Infocentros Comunitarios, en los emprendimientos sobre todo en los sectores urbano-marginales, partiendo de la hipótesis de que existe una disparidad marcada entre el sector urbano y rural en materia de acceso a las TIC, y por ende en las oportunidades de generación de emprendimientos, que se ven mitigados por el acceso y uso de los infocentros comunitarios.

Para el efecto, se ha efectuado una investigación tanto exploratoria como descriptiva a través de distintas fuentes, en su mayoría de carácter oficial, que ha permitido enfocar la situación de Ecuador en temas TIC y su incidencia en relación al nivel de emprendimiento.

\section{Metodología}

Para el efecto, se ha efectuado una investigación tanto exploratoria como descriptiva a través de distintas fuentes, en su mayoría de carácter oficial, que ha permitido enfocar la situación de Ecuador en temas TIC y su incidencia en relación al nivel de emprendimiento.

\section{Relación de las TIC con el emprendimiento}

Es importante destacar que el acceso a internet y despliegue de la banda ancha como parte esencial de las TIC, constituyen un papel crucial para favorecer y propender hacia el desarrollo económico de un país.

Precisamente, en la investigación del impacto económico de la banda ancha en América Latina (Katz, 2010), se determina que un aumento en las conexiones de banda ancha generan un efecto positivo en el crecimiento del PIB, concluyendo además que si las conexiones aumentaran $10 \%$, el PIB aumentaría 0,037\%. En resumen, en el estudio mencionado, se indica que a mayor penetración de banda ancha, mayor será el impacto de su expansión en el crecimiento del PIB. La implicancia en términos de política pública es clara: la maximización del impacto económico de la banda ancha depende de un aumento significativo de su penetración.

Puntualmente, a manera de acotar el presente análisis, se entenderá por TIC lo relacionado con conectividad y banda ancha (tanto fija como móvil), por su incidencia en el crecimiento económico y porque sobre todo le permite al emprendedor acceder a un sin número de fuentes de información global, detectar nuevas necesidades y llegar a nuevos mercados. 
Aspectos asociados a la brecha digital que existe entre países desarrollados y países en vías de desarrollo, se evidencia precisamente en el grado de emprendimiento y su relación con el bienestar económico de los países. En este contexto, surge un interés particular por la banda ancha móvil, ya que es la plataforma más dinámica para trasladar los beneficios de las TIC hacia el ecosistema emprendedor.

Un aspecto a destacarse en la banda ancha móvil por sobre la banda ancha fija, radica precisamente en la facilidad de uso, sin limitantes como el tener que contar con una computadora, ni tampoco se requiere de conocimientos técnicos avanzados. El manejo del celular como tal, y las apps en los celulares son bastante intuitivos.

Importante destacar además que de forma general en los países en vías de desarrollo el medio que ha tenido un mayor despliegue y aceptación para acceder a internet, es precisamente la banda ancha móvil. Estas ventajas permiten que el Internet esté al alcance de los emprendedores y promueven la transición de la experiencia de trabajo del escritorio al móvil.

\section{Ecuador y su situación en la región respecto a la conectividad}

De acuerdo a datos provistos por CEPAL, en su Informe referente al estado de la banda ancha en América Latina y El Caribe, se indica que el avance de Internet continúa en la región: el $56 \%$ de sus habitantes usaron la red en el año 2016, esto representa un aumento de 36 puntos porcentuales en una década.

En términos de asequibilidad, en el 2010 se requería destinar cerca de $18 \%$ de los ingresos promedio mensuales para contratar un servicio de banda ancha fija de $1 \mathrm{Mbps}$, mientras que a noviembre de 2017 esa cifra era de solo 1,2\%, estando todos los países por debajo del umbral del 5\% establecido como referencia de asequibilidad por la Comisión de Banda Ancha de las Naciones Unidas. Sin embargo y pese a estos avances, quedan pendientes problemas relacionados con la calidad y equidad en el acceso a Internet.

En términos de calidad del servicio, los 2 países mejor ubicados a nivel de Latinoamérica: Uruguay y Chile, solo tienen un $15 \%$ de sus conexiones con velocidades superiores a 15 Mbps y los peor ubicado con el 0,2\%. Como referencia, a nivel mundial, los 10 países más avanzados en esta materia superan el $50 \%$ de sus conexiones por encima de $15 \mathrm{Mbps}$.

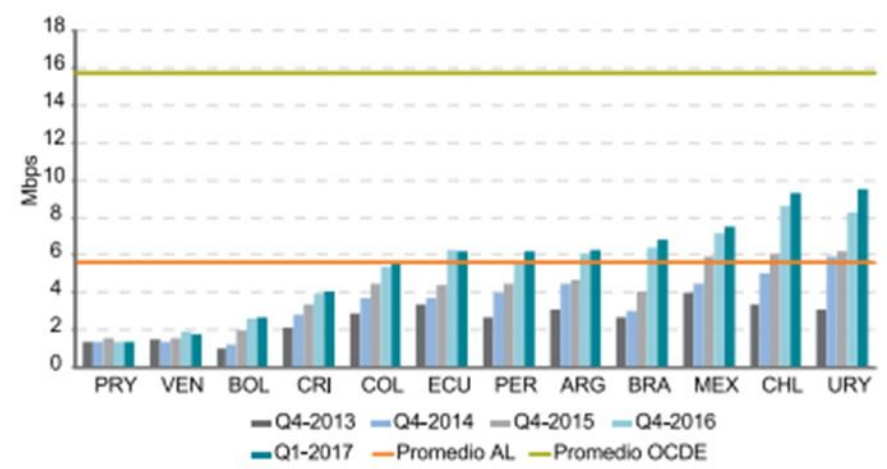

Figura 1: Evolución de las velocidades promedio efectivas de conexión mediante Banda Ancha Fija Fuente: Observatorio Regional de Banda Ancha de CEPAL, con base en State of Internet Q12017 Report 
En la Figura 2. Se tiene un comparativo regional en donde se puede observar que Ecuador ha tenido un crecimiento comparando el año 2010 y el año 2016. En cifras oficiales reportadas al INEC con corte al 2016, el porcentaje hogares que tienen acceso internet es del $36 \%$.

De este $36 \%$ de hogares que tienen acceso a internet el $31.6 \%$ usa banda ancha fija.

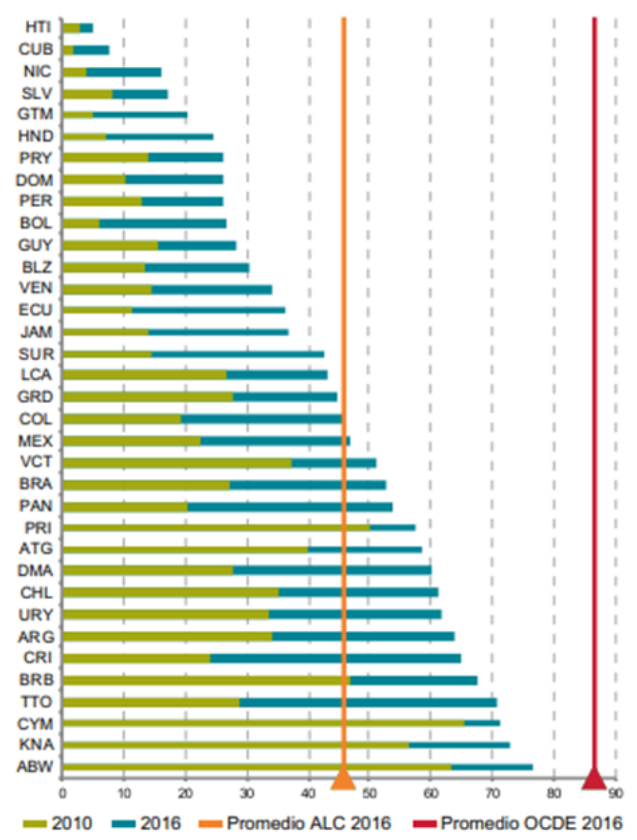

Figura 2: Porcentaje de hogares con acceso a internet por pais, 2010 y 2016

Fuente: Observatorio Regional de Banda Ancha de CEPAL, con base en datos de la UIT

En relación a la equidad, persisten las diferencias en el acceso entre las zonas rurales y urbanas, y entre quintiles de la distribución del ingreso, acentuándose lo que conocemos como brecha digital.

En el país con mayor brecha entre las áreas urbanas y las rurales, la diferencia en la penetración es de 40 puntos porcentuales y el promedio en la región es de 27 puntos porcentuales. En términos de ingresos, las brechas entre los hogares del quintil más rico en relación con el quintil más pobre llegan hasta 20 puntos porcentuales en algunos países de la región.

Por otro lado, analizando la situación de hogares con acceso a internet, desde el ámbito de quintiles poblacionales, en un comparativo regional entre 2011 y 2015, se puede observar a grandes rasgos la gran distancia existente entre el quintil 1 y quintil 5 en relación a los hogares con acceso a Internet, y observar también que a nivel regional Chile es el país que tiene una mejor cobertura. 


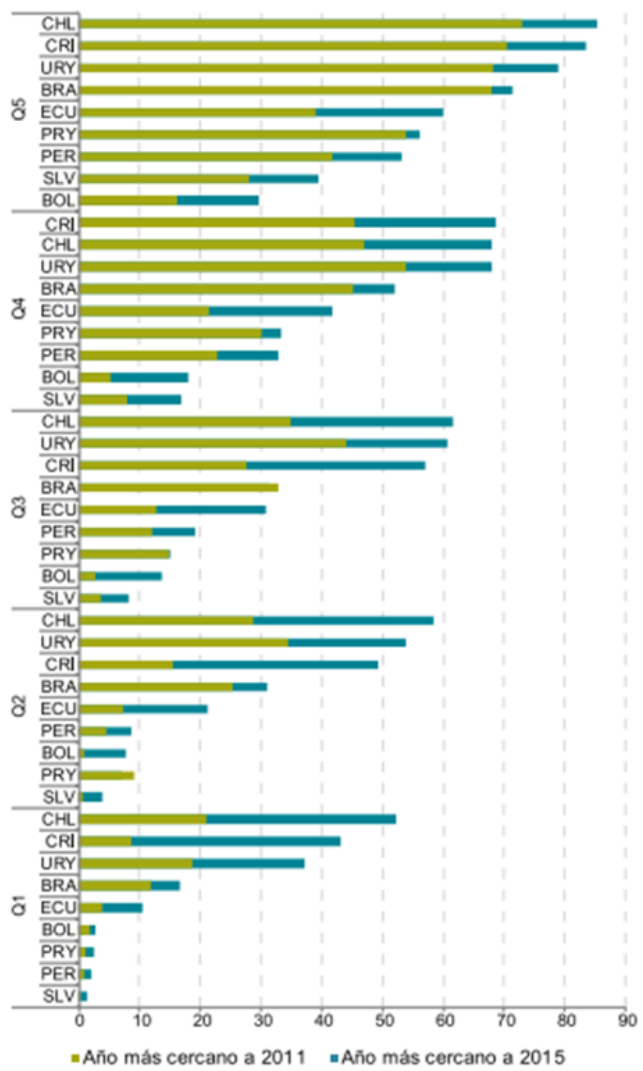

Figura 3: Porcentaje total de hogares con acceso a internet en cada quintil de ingreso Fuente: Observatorio Regional de Banda Ancha de CEPAL, con base en datos de la UIT

La penetración de Internet varía sensiblemente según las características sociodemográficas de los países. En el gráfico anterior, se muestra el porcentaje de hogares con acceso a Internet por conexión fija según quintil de ingreso. La penetración de Internet se incrementó en todos los países y quintiles entre el 2011 y el 2015.

Particularmente en Ecuador se puede observar que en este período de tiempo el porcentaje total de hogares con acceso a internet varía sustancialmente en cada quintil de la población. Si comparamos entre el quintil 1 y quintil 5, se puede observar que existe una diferencia de 50 puntos porcentuales, reflejando la disparidad acentuada por el nivel de ingresos económicos.

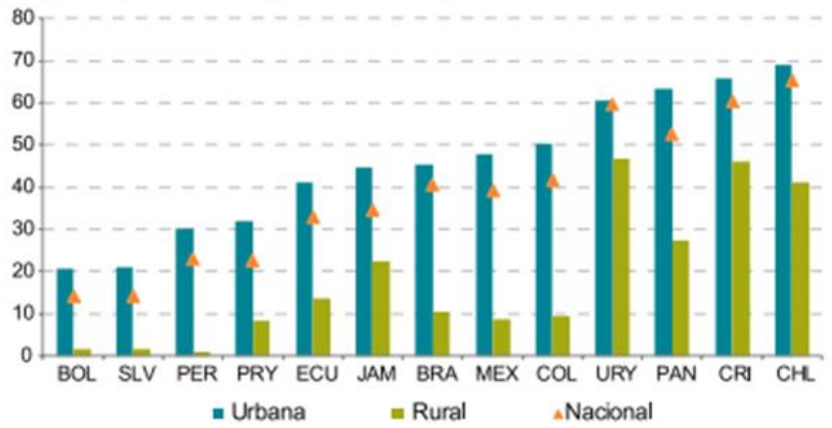

Figura 4: Porcentaje total de hogares con acceso a internet por zona urbana y rural

Fuente: Observatorio Regional de Banda Ancha de CEPAL, con base en datos de la UIT 
En la Figura 4 se muestra el porcentaje de hogares con acceso a Internet según la zona geográfica: urbana o rural, para el año 2015. En los países de la muestra, se aprecian importantes diferencias entre los medios urbano y rural. En promedio, la brecha entre ambos es de alrededor de 27 puntos porcentuales.

A nivel de Ecuador se puede observar que comparativamente con los países de la región, el porcentaje de hogares con acceso a internet está debajo de la media regional. Y específicamente si analizamos los datos a nivel de Ecuador, existe un contraste marcado entre las zonas urbana y rural.

En la Figura 4, con respecto a la penetración de banda ancha fija y móvil, con información al año 2016, se puede observar los niveles de penetración de banda ancha fija y banda ancha móvil para algunos países de América Latina y el promedio regional en 2016.

En 2010, la penetración de la banda ancha fija y la banda ancha móvil era prácticamente la misma (cerca al 6.5\%). Desde entonces, el despliegue de la banda ancha móvil sobrepasó ampliamente al de la banda ancha fija. En 2016, la banda ancha móvil al 64\% y la banda ancha fija al $11 \%$.

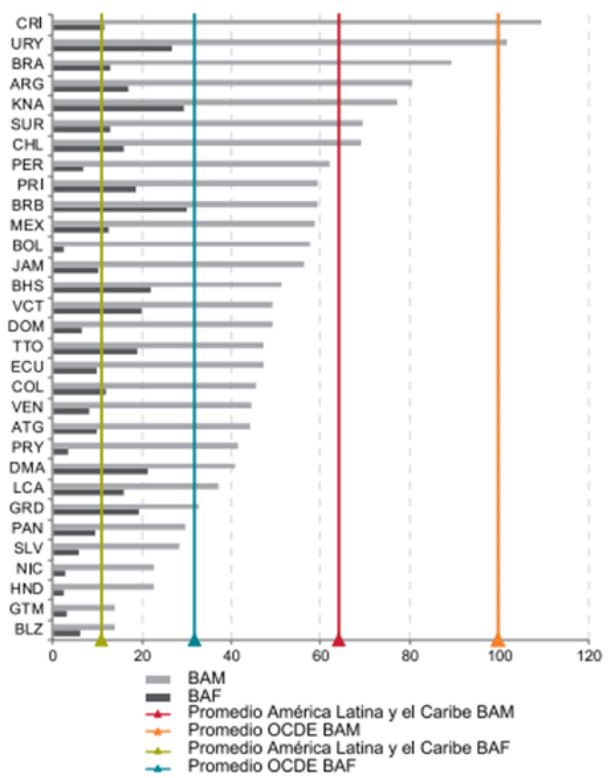

Figura 5: Penetración de banda ancha fija y móvil al 2016 (Suscripciones activas por cada 100 habitantes Fuente: Observatorio Regional de Banda Ancha de CEPAL, con base en datos de la UIT

Entre 2014 y 2016 la inserción de la Banda Ancha Móvil en Ecuador creció 16,3\%, según el Estado de Banda Ancha, de la Comisión Económica para América Latina y el Caribe (Cepal).

El estudio menciona que Ecuador contaba en 2014 con 30,9\% de penetración poblacional de este servicio; en el 2015, con el 35,09\%; y en el 2016 con 47,2\%.

Hasta agosto de 2017 líneas activas de 4G sumaban 3.5 millones, lo que representa el $23 \%$ de todas las líneas, a nivel nacional. 
En Boletín Estadístico Unificado, de ARCOTEL al mes de Junio del 2017, se indica que el 91\% de la población en el país tiene acceso al Servicio Móvil Avanzado.

No obstante, la capacidad de transmisión de datos en banda ancha fija es aún inferior en comparación a la banda ancha móvil y no hay sustitución entre ellas, sino que ambas se complementan.

\section{Posición de Ecuador respecto al Índice de Actividad Emprendedora Temprana - TEA}

Es necesario notar que Ecuador se caracteriza por tener una alta tasa de emprendimiento, y que es necesario canalizar y optimizar estos procesos iniciales productivos, con un adecuado acompañamiento y estrategias que permitan que estos emprendimientos no se vean truncados, $\mathrm{y}$ que aporten al crecimiento económico del país.

Según el Informe Global Entrepreneurship Monitor (GEM), se determinó que Ecuador obtuvo en el índice de actividad emprendedora temprana (TEA) un 31.8\%, que prácticamente señala que uno de cada tres adultos en el país ha desarrollado actividades relacionadas a crear un negocio, o en su defecto ya tenía uno que no superaba los 42 meses de existencia.

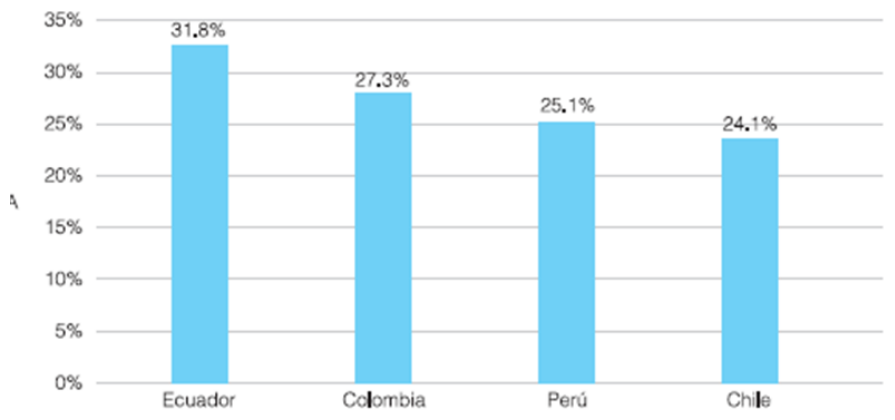

Figura 6: Países con TEA más alto en la Región

Fuente: Global Entrepreneurship Monitor Ecuador 2016

Con esto se identifica que Ecuador sigue siendo el país con el TEA más alto en la región, y el segundo entre los 66 países participantes. Sin embargo, a pesar de que en el país, se tiene un gran espíritu emprendedor, por sobre otros países en la región, se señala además en este estudio que el impacto que generan los negocios ecuatorianos en cuanto a creación de empleo es bajo, tanto así que el $72.8 \%$ de los emprendimientos son unipersonales y con bajas expectativas de generación de empleo.

Para los próximos 5 años casi un 31\% de los negocios nacientes, $48 \%$ de los nuevos y $47 \%$ de los establecidos no esperan generar ninguna plaza de trabajo. Adicional al bajo impacto de los emprendimientos en la generación de empleo, también se observa un estancamiento en la internacionalización de los negocios, donde el $92 \%$ de los emprendimientos de la TEA no tienen clientes internacionales, cifra muy por encima de países como Colombia y Chile quienes muestran tasas de 67 y 52\% respectivamente. (Global Entrepreneurship Monitor, 2016). Un $20.8 \%$ de la población adulta afirmó haber emprendido aprovechando una oportunidad, mientras que un $8.9 \%$ lo hizo por necesidad. Entre los emprendedores por oportunidad, el $22.5 \%$ lo hizo 
para mejorar ingresos actuales, el $35.2 \%$ para obtener mayor independencia y el $42.3 \%$ por ambos motivos.

En este estudio se hace un análisis respecto al índice TEA, comparando el emprendedor urbano y rural.

Aproximadamente el $64 \%$ de los emprendedores se ubican en áreas urbanas; entre quienes emprenden por oportunidad de mejora, 67,8\% también, proporción similar a la encontrada en 2015. Entre quienes emprenden por necesidad el patrón es similar, sin embargo, la proporción de emprendedores rurales por necesidad es relativamente alta y superior a la alcanzada en 2015, contraste que puede observarse en la siguiente figura:

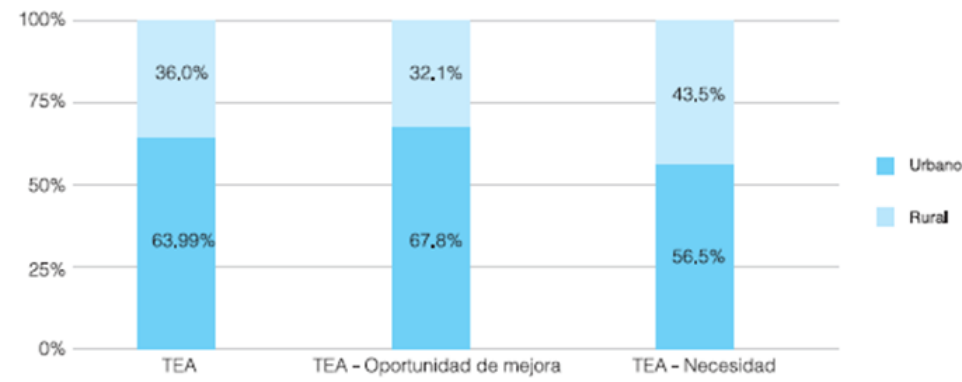

Figura 7: Composición de TEA por motivación-Contraste Urbano/Rural Fuente: Global Entrepreneurship Monitor Ecuador 2016

En este sentido, un elemento TIC sustancial como la banda ancha se torna en un aliado para reconocer y explotar oportunidades a través de emprendimientos que agreguen valor a la economía. En la medida en que estos sean aprovechados, se podrá traducir mejor la alta tasa de emprendimiento en el crecimiento económico para el país.

\section{Situación actual de los infocentros comunitarios}

Los Infocentros Comunitarios son espacios de participación y encuentro en los que se garantiza el acceso a las Tecnologías de la Información y Comunicación (TIC), contribuyendo a la reducción de la brecha digital, fomentando el desarrollo, la innovación y el emprendimiento, gracias al aprovechamiento de las TIC.

Al existir una serie deficiencia en el acceso a las TIC por parte de los sectores de la población rural, cuya situación se contrasta con la población urbana, los infocentros y su distribución geográfica, precisamente están enmarcados en mitigar esta situación dispar existente.

Actualmente, existen 852 infocentros que se encuentran distribuidos a nivel nacional en 728 parroquias beneficiando a 6.2 millones de ciudadanos, con una inversión hasta el momento de 70 millones de dólares. Se han contabilizado hasta el momento más de 12 millones de visitas y existen más de 500 mil ciudadanos capacitados en TIC. (Mintel, 2018).

Como parte los objetivos planteados que fomentan los infocentros se encuentra: 
- Capacitar en el uso de las TIC y promover emprendimientos productivos

- Promover el uso de servicios gubernamentales en línea.

- Promoción y difusión del gobierno en territorio.

Las capacitaciones que se imparten en los infocentros, son en los siguientes temas:

- Introducción a las TIC.

- TIC Negocios MiPymes.

- TIC para NIÑ@S.

- TIC Turismo.

- Redes Sociales para jóvenes.

- Herramientas de Gobierno Electrónico.

- Microempresario Digital.

- Herramientas ofimáticas.

- TIC Artesanos.

- TIC Emprendimiento.

- TIC Agricultura.

- Ensamblaje y mantenimiento de computadoras.

- Redes sociales herramientas de comunicación.

Precisamente, desde este primer objetivo consistente en la capacitación en el uso de las TIC y la promoción de emprendimientos productivos, las capacitaciones de TIC Emprendimiento y TIC Negocios MiPymes están enfocadas hacia ese segmento poblacional que busca herramientas que le ayuden en ese ámbito, debido a que los infocentros promueven el desarrollo personal, social y económico, a través de capacitaciones gratuitas, que impulsan ideas de negocio, emprendimientos y proyectos innovadores, gracias a la apropiación y correcto uso de las tecnologías, con seguimiento y apoyo para lograr casos de éxito.(MINTEL, 2018)

El pensum de TIC Emprendimiento incluye los siguientes temas:

- Emprendimiento.

- Herramientas de Gobierno Electrónico para emprendedores.

- Alternativas de Comercio Electrónico.

- Marketing Digital.

\section{Resultados}

En el contexto de este análisis, tomando en cuenta que existe una relación directamente proporcional entre el despliegue de banda ancha y en incremento en el PIB en cualquier economía, se parte de la premisa que es relevante conocer el estado de banda ancha en Ecuador. Es por tal motivo que se ha optado por establecer una relación entre el porcentaje de cuentas de internet fijo, cuyo dato se lo tiene desglosado por provincia a nivel nacional con corte al mes de marzo del 2018 (Arcotel) y analizar las diferencias en el acceso en zonas urbanas y rurales. Esto a su vez relacionarlo con la distribución de infocentros a nivel nacional y la cantidad de ciudadanos capacitados en relación a temas de emprendimiento. 
Actualmente, se puede observar que a nivel provincial, el porcentaje de acceso a internet fijo se concentra principalmente en las provincias más grandes del país, Pichincha y Guayas, seguido de la provincia de Azuay y Manabí. Las restantes 20 provincias alcanzan un 30.1\% inferior al valor alcanzado por la provincia de Pichincha.

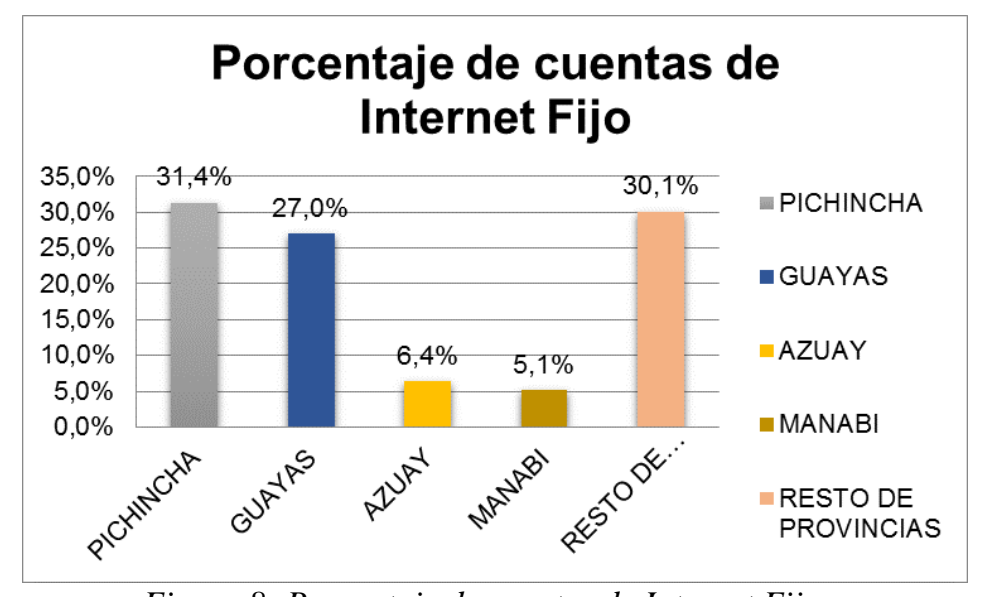

Figura 8: Porcentaje de cuentas de Internet Fijo

Fuente: Arcotel, corte Marzo 2018

El acceso a internet según el área urbana o rural evidencia la disparidad existente. De acuerdo a lo reportado por INEC en su Reporte de Tecnologías de la Información y Comunicación (TIC 2016), el 36\% de los hogares a nivel nacional tienen acceso a internet

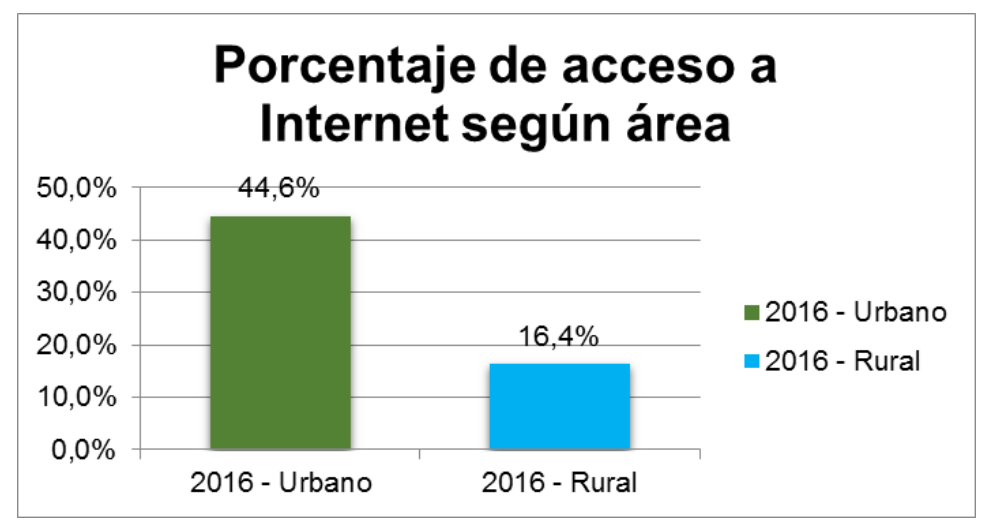

Figura 9: Porcentaje de acceso a Internet de hogares, según área urbana y rural. Fuente: INEC, corte Diciembre 2016

En tal sentido, los infocentros comunitarios, en cierto modo, son parte de la política estatal que busca disminuir la brecha digital existente entre lo urbano y rural, promoviendo entre otros aspectos el emprendimiento.

Tomando como referencia la información publicada en el sitio oficial de MINTEL, se tiene que la distribución de los infocentros a nivel nacional ordenado por provincia de mayor a menor es la siguiente: 


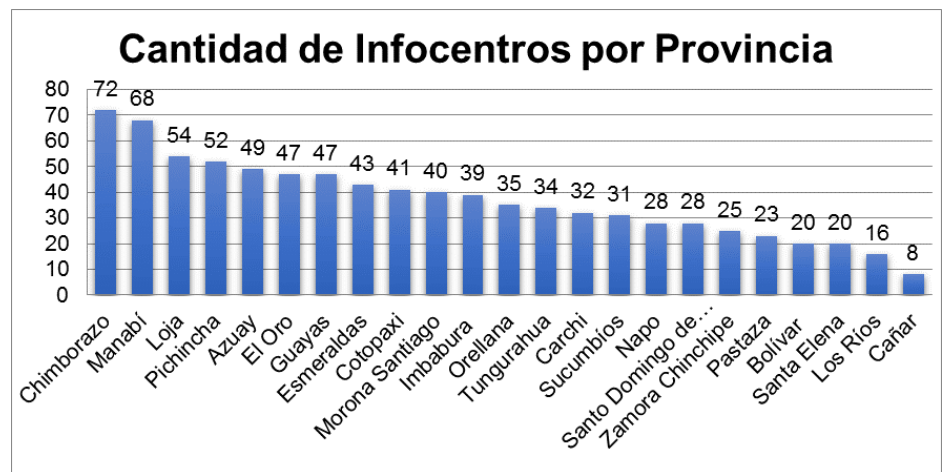

Figura 10: Cantidad de Infocentros por Provincia (2017)

Fuente: MINTEL

La mayor cantidad de infocentros se encuentran ubicados en la provincia de Chimborazo, que estadísticamente con cifras oficiales del INEC concentra una alta tasa de analfabetismo digital $(42.1 \%)$.

Del total de infocentros a nivel nacional que suman 852, existen 685 infocentros que se encuentran ubicados en la zona rural, lo que representa el $80.4 \%$.

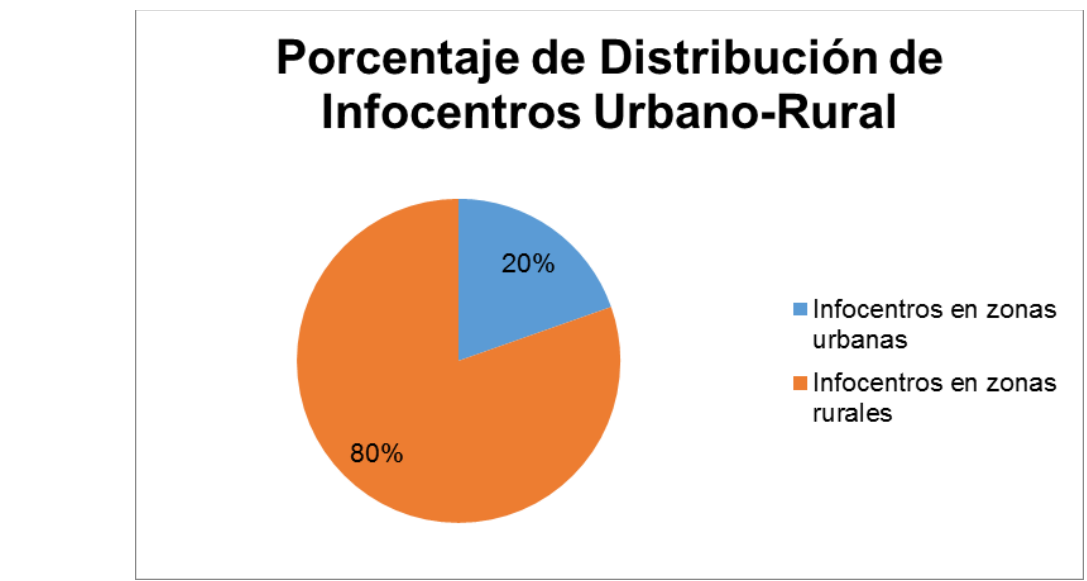

Figura 11: Porcentaje de Infocentros a nivel nacional por zona urbana y rural (2017)

Fuente: MINTEL

Con respecto a la cantidad de población capacitada en los infocentros a nivel nacional durante el año 2017, se observa que los ciudadanos ubicados en las provincias de Chimborazo, Loja, Guayas y Manabí tienen una mayor respuesta y se han beneficiado en mayor número en los infocentros ubicados en estas provincias. 


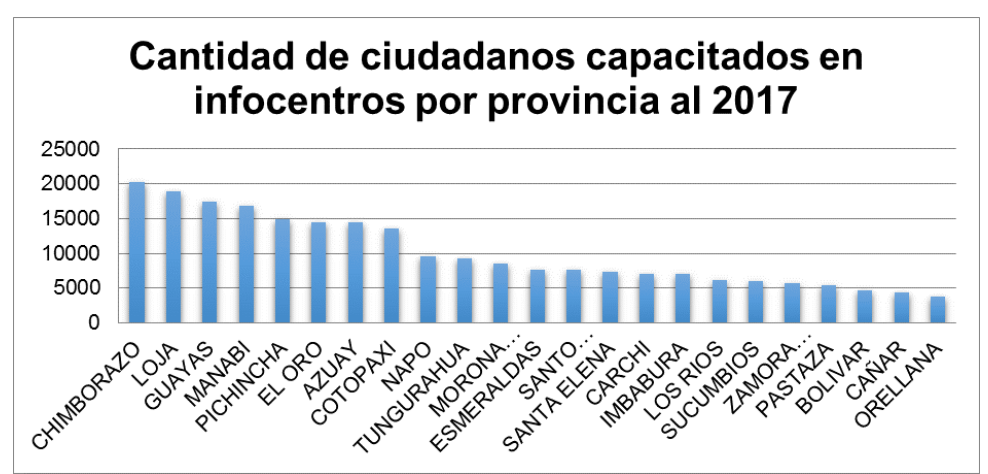

Figura 12: Cantidad de ciudadanos capacitados en infocentros por provincia al año (2017)

Fuente: MINTEL

Igualmente, del análisis se desprende que de los ciudadanos que han sido capacitados en los infocentros específicamente en temas de emprendimiento, las provincias que han tenido una mayor aceptación han sido las provincias de la región correspondiente al Oriente y la Costa, tal como se puede apreciar en la siguiente Figura.

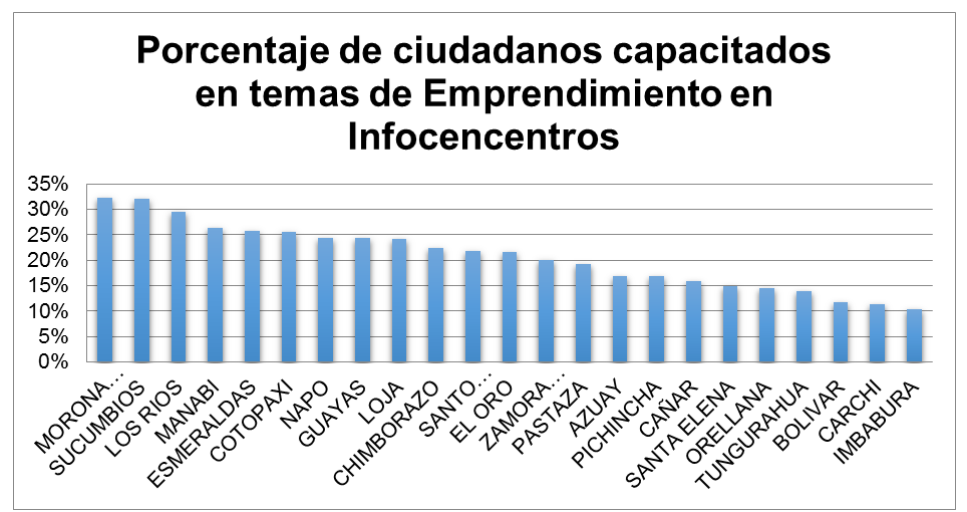

Figura 13: Procentaje de ciudadanos capacitados en temas de Emprendimiento en Infocentros (2017) Fuente: MINTEL

Haciendo un comparativo en relación a la cantidad total de ciudadanos capacitados en los infocentros, y de esta totalidad, cuántos lo hicieron en temas de emprendimiento, se puede observar que en promedio el $21 \%$ del total de las capacitaciones, se enfoca en temas de emprendimiento, que son asimilados por la ciudadanía de las diferentes provincias, nuevamente reflejando una mayor concentración en la provincia de Chimborazo, seguida por Guayas, Pichincha y Azuay, en ese orden. 


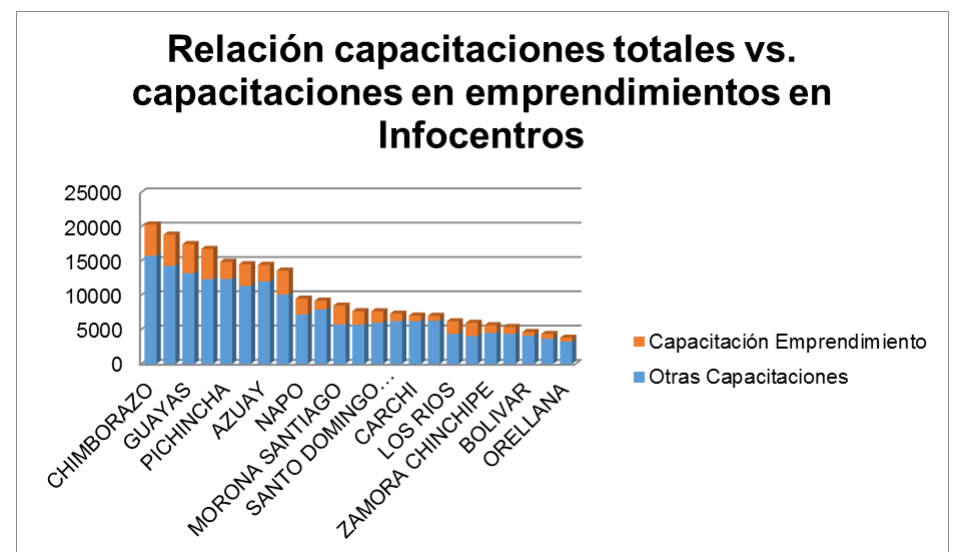

Figura 14: Porcentaje de ciudadanos capacitados en temas de Emprendimiento en Infocentros (2017) Fuente: MINTEL

Todo este proceso de capacitaciones que se vienen desarrollando en los infocentros, y más sobre todo en temas de emprendimiento, han coadyuvado a que se generen nuevas ideas de negocios, que justamente se sustentan en tener conectividad y acceso a Internet, haciendo uso de la infraestructura de los infocentros.

Los emprendedores que acceden a los infocentros han empezado a presentar y difundir sus productos en la web, utilizando el conocimiento adquirido, incursionando en un nuevo escenario, diferente al habitual, el escenario digital. Se evidencia que los emprendedores de las provincias de Azuay, Guayas, Cañar y Napo son quienes efectivamente usan los infocentros en iniciativas puntuales de emprendimiento.

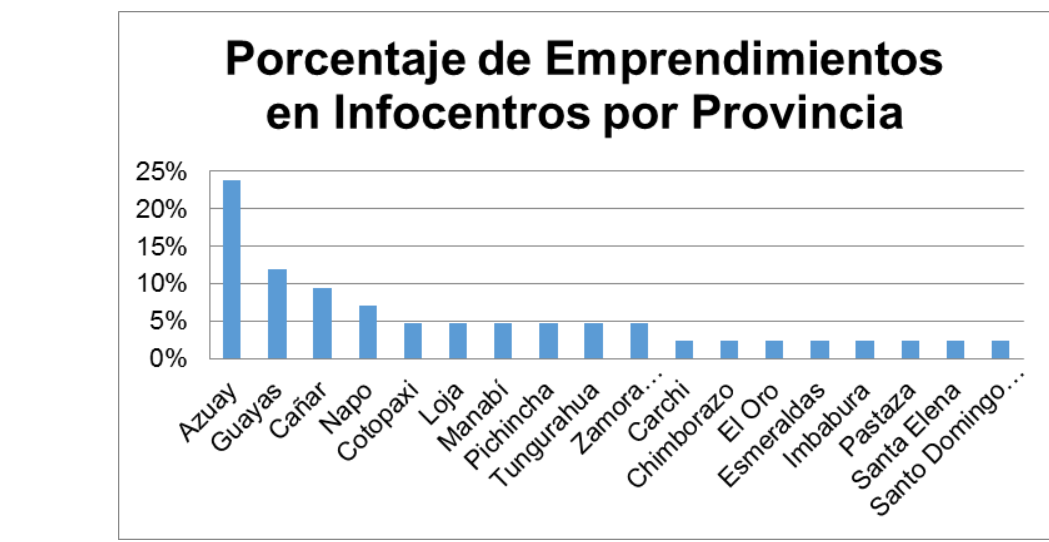

Figura 15: Porcentaje de emprendimientos en Infocentros por Provincia (2017) Fuente: MINTEL

Se corrobora además que el índice de emprendimiento temprano en Ecuador, refleja un mayor empoderamiento en el sector urbano sobre el rural. Y analizando las motivaciones para emprender, se ha establecido una categorización por oportunidad y por necesidad. En donde se puede cotejar que la ruralidad emprende en un mayor porcentaje por necesidad. En tal sentido, el rol que juegan los infocentros comunitarios en la ruralidad, es importante en el apoyo a la generación de emprendimientos. 


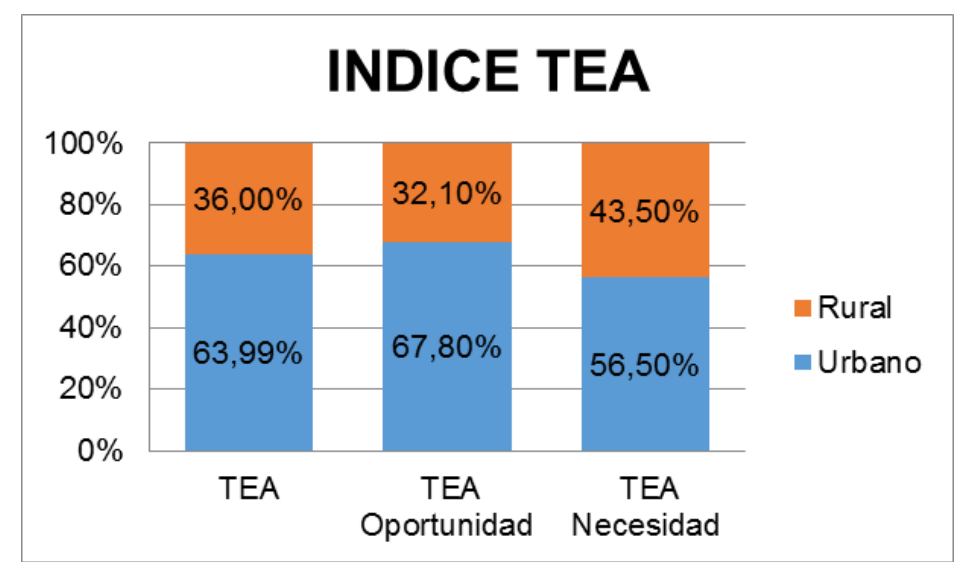

Figura 16: Índice de Emprendimiento Temprano por Oportunidad y por Necesidad en Ecuador Fuente: Global Entrepreneurship Monitor Ecuador 2016 


\section{Conclusiones}

Del análisis efectuado se puede identificar que las provincias mejor puntuadas con acceso a internet son Pichincha, Guayas, Azuay y Manabí, corroborando la hipótesis de que las oportunidades se concentran en las grandes ciudades. Así mismo se destaca la disparidad en cuanto al acceso a internet de los hogares en zonas rurales que dista mucho del acceso en zonas urbanas.

Es por este motivo que la distribución de los infocentros, cuyo énfasis es la cobertura de las zonas rurales, es una estrategia que apalanca el acceso a Internet de los sectores menos favorecidos. Las provincias de Chimborazo, Pichincha, Loja e Imbabura, son las que tienen una mayor cantidad de infocentros en zonas rurales.

En los infocentros, la ciudadanía es capacitada en múltiples temáticas, orientadas hacia la disminución de la brecha digital, entre las que se destacan la capacitación en temas de Emprendimiento y TIC, con una mayor aceptación en las provincias de Chimborazo, Guayas, Pichincha y Azuay.

Las provincias que han acogido de mejor manera las capacitaciones recibidas, y con ese conocimiento han generado nuevas ideas de negocios han sido: Azuay, Guayas, Cañar y Napo.

Es importante resaltar que las capacitaciones en materia de emprendimiento, que se brindan en los infocentros a nivel nacional han servido de semillero para que surjan nuevas ideas de negocio, facilitando que los emprendedores puedan dar a conocer sus productos hacia nuevos mercados, concientizándose de que el acceso a Internet y todos los recursos TIC asociados les permiten superar barreras que en un escenario tradicional, probablemente no les sería posible.

Se corrobora además que en los sectores rurales, los emprendimientos surgen más con un sentido de necesidad que de oportunidad. Precisamente aquí radica la importancia del despliegue existente de los infocentros comunitarios a nivel nacional, posibilitando el acceso a las TIC de los sectores menos favorecidos de la población, ubicados sobre todo en zonas rurales, y la contribución de los mismos hacia la generación de emprendimientos a través de las TIC.

\section{Bibliografía}

Acz, Z., L. Szerb E. Autio, y A. Lloyd (2016). Global Entrepreneurship Index 2017. The Global Entreprenurship and Developmet Institute, Washington DC USA.

Cáceres, P., (2017). Banda ancha móvil: ¿Aliada de emprendedores y del desarrollo económico del país? Recuperado: https://www.esan.edu.pe/conexion/actualidad/2017/08/14/bandaancha-movil-aliada-de-emprendedores-y-del-desarrollo-economico-del-pais/

Rojas, E. (2012). Conectados a la Banda Ancha: Tecnología, políticas e impacto en América Latina y España. Recuperado: https://repositorio.cepal.org/bitstream/handle/11362/4017/1/S2012101_es.pdf 
CEPAL, (2018). Estado de la Banda Ancha en América Latina y EL Caribe 2017, recuperado https://repositorio.cepal.org/bitstream/handle/11362/43365/1/S1800083_es.pdf

Cornell University, INSEAD, and WIPO (2016): The Global Innovation Index 2016: Winning with Global Innovation, Ithaca, Fontainebleau, and Geneva.

Caicedo, G., Lasio, V., Ordeñana, X., Samaniego, A., Zambrano, J. (2017). Global Entrepreneurship Monitor Ecuador 2016, recuperado: http://www.espae.espol.edu.ec/wpcontent/uploads/2017/06/ReporteGEM2016.pdf

Instituto Nacional de Estadísticas y Censos, INEC. (2012). VII Censo de Población y Vivienda en el Ecuador.

Instituto Nacional de Estadísticas y Censos, INEC. (2016). Encuesta Nacional de Empleo, Subempleo y Desempleo - ENEMDU, realizada mediante uso del Software de escritorio "Observatorio Económico Social"

Instituto Nacional de Estadísticas y Censos, INEC. (2016). Tecnologías de la Información y $\begin{array}{llll}\text { Comunicación } & \text { (TIC) } & -2016 . & \text { Recuperado de }\end{array}$ http://www.ecuadorencifras.gob.ec/documentos/webinec/Estadisticas_Sociales/TIC/2016/170125.Presentacion_Tics_2016.pdf

Katz, R. y F. Callorda (2011). Medición de Impacto del Plan Vive Digital en Colombia y de la Masificación de Internet en la Estrategia de Gobierno en Línea, Centro de Investigación de la Telecomunicaciones (CINTEL), Bogotá, Colombia, diciembre.

Katz, R. (2009), El papel de las TIC en el desarrollo: Propuesta de América Latina a los retos económicos actuales. Colección Fundación Telefónica, Madrid: Ariel.

Kelley, D., Herrington, M. \& Singer, S. (2017). Global Entrepreneurship Monitor: 2016/17 GLOBAL REPORT. Global Entrepreneurship Research Association (GERA) 2016.

Sanguinetti, P. (2013). El emprendimiento como motor de desarrollo. Caracas: CAF.

UIT, (2011). La Banda Ancha y la Economía: Crecimiento, Productividad y Empleo. Recuperado de https://www.itu.int/net/itunews/issues/2011/05/pdf/201105_14-es.pdf

Kappler, L., Amit, R., Guillén, M. y Qusada, J. M. (2007). Entrepreneurship and Firm Formation Across Countries. The World Bank Development Research Group Finance and Private Sector Team. Policy Research Working Paper 4313.

MINTEL, (2018), Estadísticas Infocentros. Recuperado de: https://infocentros.mintel.gob.ec/estadisticas-infocentros/

PROECUADOR (2016). Boletín de Comercio Exterior noviembre-diciembre 2016, Tomado de http://www. proecuador.gob.ec/pubs/proec_ic_06_71/ 
Roller, L-H. Y L. Waverman (2001), "Telecommunications Infrastructure and Economic Development: A simultaneous approach", American Economic Review, 91(4), pp. 909-23.

Vergara, A. (2011), Aplicación del análisis tecno-económico al despliegue de redes de acceso de próxima generación. El caso de la competencia entre plataformas, la regulación y las políticas públicas en España, tesis doctoral, UPM. Disponible en: http://oa.upm.es/9837/

World Economic Forum (2012), Maximizing the Impact of Digitization, Global Information Technology Report (GITR). 